\title{
Prechemotherapy subclinical left ventricular dysfunction: Do we know the mechanism?
}

We would like to thank $\mathrm{Dr}$ Tadic and colleague for their interest in our research manuscript titled "Pediatric Malignancies: Is the Pre-chemotherapy Left Ventricular Function Normal?"1 and their contribution in the research related to chemotherapy-induced cardiomyopathy. Tadic et al have recently published their research findings of abnormal left ventricular (LV) systolic function in 122 chemotherapy naïve and radiotherapy naïve cancer patients (prechemotherapy) and 45 age-matched controls. They reported significant abnormality in LV systolic function in cancer patients compared to controls and reported a decrease in LV global longitudinal strain, (19.1 $\pm 2.1 \%$ vs $17.8 \pm 3.5 \%, P-.022)$, LV global circumferential strain, $(22.9 \pm 3.5 \%$ vs $20.1 \pm 4.1 \%, P<.001)$, and LV global radial strain, $(40.5 \pm 8.8 \%$ vs $35.2 \pm 10.7 \% ; P-.004){ }^{2}$ These finding are similar to findings in our study population where we have found significant abnormalities LV systolic function as assessed by speckle strain imaging in prechemotherapy group compared to normal group. However, there were no statistical significant differences in LV systolic function on subgroup analysis of solid vs. hematological tumors.

Finding of LV systolic function in this fragile population even before use of chemotherapy is very intriguing. The mechanism of the underlying abnormalities in the left ventricular dysfunction, as assessed by GLS, in the cancer patients is not completely studied. So far the mechanisms of cancer leading to subtle changes in LV systolic function even before the use of chemotherapy are speculative. Several mechanisms have been postulated including the effect of anemia, higher inflammatory state, and direct infiltration of myocardium with malignant cells in cancer patients. The effect of anemia on LV systolic function and strain was evaluated by Zhou et al in noncancer patients. The 39 adult patients with hemoglobin $(\mathrm{Hb}) 6-9 \mathrm{~g} / \mathrm{dL}$ had abnormal GLS $(-18.3 \pm 1.8$ vs $-20.8 \pm 2.6$ vs $-21.4 \pm 2.7)$, abnormal GCS (-18.1 \pm 3.7 vs $-19.6 \pm 4.6$ vs $-19.8 \pm 4.2)$, higher LV end-diastolic volume (115.0 \pm 27.4 vs $97.2 \pm 29.4$ vs $94.9 \pm 31.7)$, higher LV end-systolic volume ( $40.2 \pm 9.9$ vs $36.9 \pm 10.1$ v. $35.7 \pm 12.3$ ), and higher LV indexed mass ( $77.4 \pm 6.8$ vs $73.8 \pm 7.9$ vs $72.5 \pm 8.2$ ) as compared those with 44 age-matched adults with $\mathrm{Hb}>9 \mathrm{~g} / \mathrm{dL}$ and 40 matched healthy volunteers. ${ }^{3}$ Among our population of pediatric cancer patients, the patients with leukemia had significant lower $\mathrm{Hb}$ compared to the group with solid tumors $(\mathrm{Hb} 8.4 \pm 3.3$, $\left.11.4 \pm 3.6 P<.001^{*}\right)$. However, the LVGLS was equally decreased in both groups of patients $(-19.5 \pm 2.4 \mathrm{vs}-19.2 \pm 2.9, P-.7) .{ }^{1}$ There is another proposed theory of infiltration of the myocardium by circulating cancer cells leading to cardiac dysfunction. Leukemic infiltrates and hemorrhage were observed in $69 \%$ of the 420 hearts in an autopsy study of leukemia patients. ${ }^{4}$ The proposed mechanism of heightened inflammatory state in malignancies and association with changes in the LV systolic function is not completely understood. In an animal study, GLS was decreased in dogs with systemic inflammatory response syndrome compared to healthy dogs $(-14.6 \pm 3.2$ vs $-18.5 \pm 4.1 ; P-.003) .^{5}$ In a meta-analysis of 8 studies involving 794 patients, there was a significant association between the worse LV function and GLS values and mortality (standard mean difference -0.26 ; $95 \% \mathrm{Cl}-0.47,-0.04, P-.02$ ) in patients with severe sepsis/ and or septic shock. There was no association between LVEF and mortality in the same population of patients. ${ }^{6}$ Therefore, the mechanism leading to prechemotherapy changes in the LV systolic function is still elusive.

To study the underlying mechanism of prechemotherapy LV dysfunction in patients with malignancies, larger studies in a prospective model designed to evaluate the left ventricular function and their correlation with the cardiac biomarkers and biochemical markers of inflammation are warranted. This calls for a collaborative effort between with the cardiologist and the oncologist. As a first step, it is important to recognize that prechemotherapy LV systolic dysfunction exists, which can be detected by the speckle tracking echocardiography. As more and more research data are being published, we propose adding the speckle echocardiography for assessing the LV systolic function in patients even before they have received chemotherapy. This may help stratify high-risk patients who are at risk for chemotherapy and radiotherapy induced cardiotoxicity.

Jyothsna Akam-Venkata MBBS ID
Gilda Kadiu RDCS
James Galas MD
Sanjeev Aggarwal MD

Division of Cardiology, Carman and Ann Adams Department of Pediatrics, Children's Hospital of Michigan, Wayne State University School of Medicine, Detroit, Michigan

Correspondence

Jyothsna Akam-Venkata, MBBS, Pediatric Cardiology Fellow, The Carman and Ann Adams Department of Pediatrics, Wayne State University School of Medicine, Children's Hospital of Michigan, 3901 Beaubien Boulevard, Detroit,

MI 48201.

Email: jyothsnaav@gmail.com 


\section{ORCID}

Jyothsna Akam-Venkata iD https://orcid.org/0000-0001-8625-6020

\section{REFERENCES}

1. Akam-Venkata J, Kadiu G, Galas J, Aggarwal S. Pediatric malignancies: Is the prechemotherapy left ventricular function normal? Echocardiography. 2019;36(9):1727-1735.

2. Tadic M, Genger M, Baudisch A, et al. Left ventricular strain in chemotherapy-naïve and radiotherapy-naive patients with cancer. Can J Cardiol. 2018;34(3):281-287.

3. Zhou Q, Shen J, Liu Y, Luo R, Tan B, Li G. Assessment of left ventricular systolic function in patients with iron deficiency anemia by three-dimensional speckle-tracking echocardiography. Anatol J Cardiol. 2017;18(3):194-199.

4. Roberts WC, Bodey GP, Wertlake PT. The heart in acute leukemia. A study of 420 autopsy cases. Am J Cardiol. 1968;21(3):388-412.

5. Corda A, Pinna Parpaglia ML, Sotgiu G, et al. Use of 2-dimensional speckle-tracking echocardiography to assess left ventricular systolic function in dogs with systemic inflammatory response syndrome. J Vet Intern Med. 2019;33(2):423-431.

6. Sanfilippo F, Corredor C, Fletcher N, et al. Left ventricular systolic function evaluated by strain echocardiography and relationship with mortality in patients with severe sepsis or septic shock: a systematic review and meta-analysis. Crit Care. 2018;22(1):183. 